Canadian

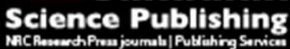

Applied Physiology, Nutrition, and Metabolism Physiologie appliquée, nutrition et métabolisme

\title{
Differential contribution of visual and auditory information to accurately predict the direction and rotational motion of a visual stimulus
}

\begin{tabular}{|r|l|}
\hline Journal: & Applied Physiology, Nutrition, and Metabolism \\
\hline Manuscript ID & apnm-2015-0390.R2 \\
\hline Manuscript Type: & Article \\
\hline Date Submitted by the Author: & 05-Nov-2015 \\
\hline Complete List of Authors: & $\begin{array}{l}\text { Park, Seoung Hoon; University of Florida, Department of Applied and } \\
\text { Physiology } \\
\text { Kim, Seonjin; Seoul National University } \\
\text { Kwon, MinHyuk; University of Florida } \\
\text { Christou, Evangelos; University of Florida, Department of Applied } \\
\text { Physiology and Kinesiology; University of Florida, Department of Physical } \\
\text { Therapy }\end{array}$ \\
\hline Keyword: & \begin{tabular}{l} 
visual and auditory information, prediction, direction, rotational motion \\
\hline
\end{tabular} \\
\hline
\end{tabular}

\section{SCHOLARONE}

Manuscripts 
Differential contribution of visual and auditory information to accurately predict the direction and rotational motion of a visual stimulus

Seoung Hoon Park, Seonjin Kim, MinHyuk Kwon, and Evangelos A.

Christou

Correspondence:

Seoung Hoon Park, M.S.

Department of Applied Physiology and Kinesiology

University of Florida

Gainesville, FL 32611, USA

Phone: 352-394-1750

FAX: 352-392-5262

E-mail:shpark@ufl.edu

Seoung Hoon Park, MinHyuk Kwon, and Evangelos A Christou. Department of Applied

Physiology and Kinesiology, University of Florida, Gainesville, FL 32611, USA

Seonjin Kim. Department of Physical Education, Seoul National University, Seoul, 151-748, Korea 


\section{ABSTRACT}

Vision and auditory information are critical for perception and enhance the ability of an individual to respond accurately to a stimulus. However, it is unknown whether visual and auditory information contribute differentially to identify the direction and rotational motion of the stimulus. The purpose of this study was to determine the ability of an individual to accurately predict the direction and rotational motion of the stimulus based on visual and auditory information. In this study, we recruited nine expert tabletennis players and used table-tennis service as our experimental model. Participants watched recorded services with different levels of visual and auditory information. The goal was to anticipate the direction of the service (left or right) and the rotational motion of service (topspin, sidespin, or cut). We recorded their responses and quantified the following outcomes: 1) directional accuracy; 2) rotational motion accuracy. The response accuracy was the accurate predictions relative to the total number of trials. The ability of the participants to predict the direction of the service accurately increased with additional visual information but not with auditory information. In contrast, the ability of the participants to predict the rotational motion of the service accurately increased with the addition of auditory information to visual information but not with additional visual information alone. In conclusion, this finding demonstrates that visual information enhances the ability of an individual to accurately predict the direction of the stimulus, whereas additional auditory information enhances the ability of an individual to accurately predict the rotational motion of stimulus.

\section{Key words: visual and auditory information, prediction, direction, rotational}

\section{$\underline{\text { motion }}$}




\section{INTRODUCTION}

Perception is essential for many activities of daily living. Visual information is critical in perceiving hazards and initiating a plan to avoid them, such as responding to an unexpected stimulus while driving (Deery 2000). Auditory information can supplement visual information and enhance the ability to accurately react to an unexpected stimulus. For example, the sound made by a fallen box from the leading truck may provide information about its weight, which could not be determined based on visual information alone. Therefore, visual and auditory information are critical in enhancing the ability of an individual to respond accurately to a stimulus (Welch et al. 1986; Vroomen et al. 2000; Meyer et al. 2001; Frassinetti et al. 2002; Soto-Faraco et al. 2005; Noesselt et al. 2008; Olivers et al. 2008; Lewis et al. 2010). However, it is unknown whether visual and auditory information contribute differentially to identify the direction and rotational motion of the visual stimulus. In this study, we examine this concept by using prediction of table-tennis service as an experimental model.

Visual information enables humans to respond accurately to a stimulus. The direction (Underwood et al. 2002), shape, and rotational motion (Lewis and Noppeney 2010) of the visual stimuli is discriminated by visual information. In addition, visual information is so critical for stimulus accuracy that the brain initiates the response by moving the eyes in the predicted location of the stimulus (Saunders et al. 2003). Although these studies are important in understanding the use of visual information for response accuracy, they are limited because they incorporate simple visual sequences as a stimulus such as graphical images or patterns. In real life, the visual stimulus may be more complicated and visual information may come from the complicated context of 
the stimulus. It remains unclear whether visual information contributes to identify the direction and rotational motion of a complicated visual stimulus.

Audition also provides critical perceptual information and can enhance the ability to respond accurately to a stimulus (Buchtel et al. 1996). The brain combines information from different senses to respond to a stimulus and this combined information enables us to better perceive a stimulus (Desantis et al. 2014). Auditory information is integrated with visual information to increase the ability to detect a visual stimulus (Vroomen and Gelder 2000). However, there are no studies that examined the specific advantage of combining auditory and visual information to enhance the accuracy of detecting the direction and rotational motion of a complex visual stimulus.

The purpose of our study, therefore, was to determine the ability of an individual to accurately predict the direction and rotational motion of a complex visual stimulus based on visual and auditory information. We hypothesized that visual information and added auditory information to visual information would differentially enhance the accuracy of predicting the direction and rotational motion of the stimulus. In this study, we used table-tennis service as a model to understand the contribution of visual and auditory information on prediction accuracy for direction and rotational motion.

\section{MATERIALS AND METHODS}

\section{Participants}

Nine expert table-tennis players $(22.0 \pm 2.1$ years, university male athletes experienced over 10 years) volunteered to participate in this study. They were classified as experts according to Ericsson et al. (1993) that suggested that expert performance is 
actually the result of intense practice extended for a minimum of 10 years. All participants were right-handed players and reported being healthy without any hearing disorders (maximal audio frequency $16.7 \pm 0.5 \mathrm{kHz}$ ) or uncorrected visual impairments. All procedures for this study were approved by Seoul National University, and participants gave written informed consent before participation in this study.

\section{Experimental protocol}

Each participant performed one experimental session that lasted approximately an hour. Prior to the experimental session, each participant was familiarized with the tasks and procedures of the study. The familiarization period included a verbal explanation of the task and 10 practice trials. After the familiarization period, each participant completed 60 more trials, which were composed of different levels of visual and auditory information.

Experimental conditions (ECs). Participants anticipated the direction and rotational motion of service after they watched the video clip (played in a random order; 30 frames per sec) of table-tennis services. To standardize the service form, every service in the video clips was performed by a professional male player with a pen-hold grip (a pen-hold grip or shake-hand grip mostly used; right-handed, rubber thickness: 2.2mm). The video clips were edited (Vegas Pro 12, Sony Creative Software Inc., Middleton, Wisconsin, USA) based on the following experimental conditions (Figure 2). There were four visual information conditions with exclusive visual information: 1) video ended 4 frames before impact (EC1); 2) video ended 2 frames before impact (EC2); 3) video ended at impact (EC3); 4) video ended 2 frames after impact (EC4). There was a 
single visual and auditory information condition (EC5). During this condition, participants watched a video that ended at impact (identical to EC3) but contained the sound of the racquet hitting the ball (impact sound). The reason why we added auditory information to only EC3 was that the auditory information only occurs when a racquet hits a ball. However, we did not use EC4 with auditory information because we found in a preliminary study that participants almost accurately predicted the direction with only visual information under EC4 (not suitable to assess the ability to predict the direction of a stimulus). In addition, we did not use an isolated auditory condition (no visual information) because in a preliminary study we found that when participants received only auditory information their ability to predict the direction or rotational motion of the service was not significantly different from chance. The goal of each participant was to predict the direction (2 possible directions; left or right) and rotational motion of service (3 possible rotational motions; topspin, sidespin, or cut).

\section{Experimental arrangement}

Experimental setup and apparatus. Participants were seated comfortably in the upright position and faced a 134 inch screen located 3 meters away from the participant (Figure 1). The video of the service was projected (VPL-HW30ES, resolution: $1920 \times 1080 \times 3$ pixels, brightness: 1300 lumen, Sony, Tokyo, Japan) on the screen. All participants affirmed that they could see the display clearly. In one condition, the sound of the racquet hitting the ball was provided via a speaker. The loudness of sound was approximately $50 \mathrm{~dB}$ (measured from $30 \mathrm{~cm}$ away; 407730 Digital Sound Level Meter, Extech Instruments, Nashua, New Hampshire, USA). 
Prediction. Participants indicated the expected direction and rotational motion of service using a keyboard, which was located on a desk in front of them. On the keyboard, there were two keys for direction (' $\leftarrow$ ' for left, ' $\rightarrow$ ' for right; arrow keys located between letters and numbers) and three keys for rotational motion (' $T$ ' for topspin, ' $\mathrm{S}$ ' for sidespin, 'C' for cut). Except for the five keys above, all other keys were removed to help the participants make a prompt prediction. Participants were required to make a prediction in the order of direction and rotational motion of service within $3 \mathrm{~s}$ following the end of the video clip. We provided feedback on overall response accuracy after they finished all trials.

\section{Data and statistical analysis}

Data were analyzed off-line using custom-written programs. We analyzed all 60 trials for each participant (5 experimental conditions and 12 trials at each condition). The dependent variable for this study was prediction accuracy, which was quantified for the direction and rotational motion of service. Specifically, we calculated the number of correct predictions relative to the total number of trials given for each condition. Thus, prediction accuracy is given as a percentage.

We used a one-way ANOVA to compare prediction accuracy for direction and rotational motion of service across four different experimental conditions (EC1, EC2, EC3, and EC4). Significant one-way ANOVAs were followed with post-hoc analysis (Tukey's multiple comparisons). We used a paired t-test to compare the prediction accuracy for direction and rotational motion of service between the visual information (EC3) and visual and auditory information condition (EC5). 
Analyses were performed with the IBM SPSS Statistics 21.0 statistical package (IBM Corp., Armonk, New York, USA). The alpha level for all statistical tests was 0.05. Data are reported as mean $\pm S D$ in the text and mean \pm standard error of the mean (SEM) in the figures.

\section{$\underline{\text { RESULTS }}$}

Amount of visual information and prediction of direction and rotational motion of service

We quantified prediction accuracy of direction and rotational motion of service by calculating the number of accurate predictions relative to the total number of trials. All participants performed significantly better than chance level (50\% for direction, $33.3 \%$ for rotational motion; EC1: $p<0.03$, EC2-4: $p<0.001$ ) except for one participant's prediction accuracy for direction in a single experimental condition (EC1). There was a significant one-way ANOVA for visual information conditions on directional accuracy $\left(F_{3,32}=11.853 ; p<0.001 ;\right.$ Figure 3A). Post-hoc analysis indicated that participants exhibited less directional accuracy with the experimental condition 1 ( 4 frames before impact; $67.59 \pm 18.84 \%$ ) compared with the other three experimental conditions (2 frames before impact, impact, and 2 frames after impact; 86.11 $\pm 13.18,95.37 \pm 6.05$, $98.15 \pm 3.67 \% ; p<0.05)$. Although the directional accuracy increased with more visual information (Figure 3A), there was no statistical difference among the experimental conditions of 2 frames before impact, impact, and 2 frames after impact $(p>0.2)$. The one-way ANOVA for visual information conditions on rotational motion accuracy (EC1 - 
4: $57.41 \pm 11.37,65.74 \pm 11.37,59.26 \pm 15.28,63.89 \pm 19.98 \%)$ was not significantly different $\left(F_{3,32}=0.612 ; p=0.612 ;\right.$ Figure 3B $)$.

\section{Visual and auditory information to predict direction and rotational motion of service}

We compared the prediction accuracy of direction and rotational motion of service for a visual information only condition (impact; EC3) with a visual + auditory information condition (added impact sound; EC5). All participants performed significantly better than chance level $(p<0.001)$. There was a significant difference for the rotational motion of service $(E C 3=59.26 \pm 15.28 \%$, EC5 $=75.00 \pm 12.50 \% ; t=-4.875$, $\mathrm{df}=8 ; p<0.01$; Figure 4B) but not the direction of service $(\mathrm{EC} 3=95.37 \pm 6.05 \%$, $E C 5=98.15 \pm 3.67 \% ; t=-1.414, \mathrm{df}=8 ; p>0.3 ;$ Figure $4 \mathrm{~A}$ ) between the two conditions. Specifically, participants were approximately $15 \%$ more accurate in predicting the rotational motion of service when auditory information was added to visual information.

\section{DISCUSSION}

In this paper, we asked whether visual and auditory information contribute differentially to identify the direction and rotational motion of a complex visual stimulus. We demonstrate that additional visual information can enhance the ability to identify the direction of a visual stimulus, whereas added auditory information to visual information can enhance the ability to identify the rotational motion of a visual stimulus. These results provide novel evidence that the identification of the direction of a complex visual stimulus is enhanced as the amount of visual information increases. Adding auditory information to visual information becomes critical for the identification of the rotational 
motion of a complex visual stimulus. These findings, therefore, significantly contribute to our understanding of the role of sensory information for the identification of a complex stimulus motion.

\section{Additional Visual Information}

Our results demonstrate that additional visual information can enhance the ability to identify the direction of a complex visual stimulus. This result is consistent with previous findings in different situations (Abernethy et al. 1987; Underwood et al. 2002). For example, the study by Abernethy and Russell (1987) indicated that the prediction accuracy for the direction of a visual stimulus increases with visual information in a racquet sport (badminton). Furthermore, sufficient amount of visual information is needed for drivers to scan trajectories of other vehicles on a road (Underwood et al. 2002). Identifying visual information for direction is likely related to direction specific neuronal firing in the middle temporal area (cortical areas along the dorsal pathway of the visual system) (Treue et al. 2000; Hol et al. 2001) and such neurons discharge at a higher frequency in relation to a visual stimulus in a particular direction (Georgopoulos et al. 1982). In conclusion, these findings demonstrate that humans need adequate amount of visual information to accurately predict the direction of a stimulus, and this visual processing is associated with the neurons in the visual cortex.

Results from the current study support previous findings and extend the literature by providing evidence that visual information can enhance the ability to identify the direction of a complex visual stimulus. Interestingly, there was no significant difference in the prediction accuracy for the direction of a visual stimulus above a certain amount 
of visual information (EC2 - EC3 - EC4; $p>0.2$; Figure 3A), which indicates that a certain amount of visual information is sufficient to accurately predict the direction of a complex stimulus. These findings indicate that humans are able to accurately predict the direction of a complex visual stimulus although visual information is impaired to a certain degree.

\section{Additional Auditory Information}

The most important finding in this study was that added auditory information to visual information can enhance the ability to identify the rotational motion of a complex visual stimulus. As mentioned above, sufficient amount of visual information can enhance the ability to predict the direction of a visual stimulus. Additionally, there was no significant difference in the prediction accuracy for the direction of a visual stimulus

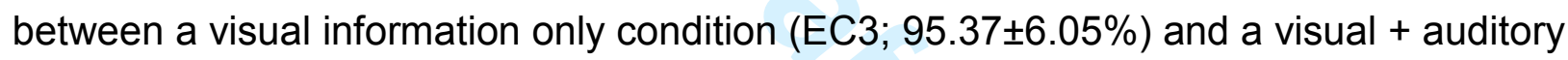
information condition (EC5; $98.15 \pm 3.67 \%$ ). It argues that visual information (above a certain amount) is sufficient to estimate the direction of a visual stimulus and no auditory information is required. However, we are not always able to acquire enough visual information to identify the direction of a stimulus in our daily life. Rather, we sometimes have to identify a complicated visual stimulus motion as correctly as possible with insufficient amount of visual information. For example, pedestrians can identify the proximity of vehicles by sound when visual information is impaired, such as in heavy fog. This situation demonstrates that in the absence of vision, the sound from a car could offer significant information to the pedestrian about the movement trajectory of the car. 
The results from our study demonstrate that the rotational motion of a visual stimulus cannot be accurately identified with only visual information. Auditory information supplements visual information and enhances the ability to accurately identify the rotational motion of a complex visual stimulus. This result is consistent with findings from previous studies (Gibson 1957; Vroomen and Gelder 2000; Lippert et al. 2007). In a study by Gibson (1957), it was found that visual and auditory stimuli provide information about the swiftness of the moving objects. The work of Vroomen and de Gelder (2000) and Lippert et al. (2007) suggested that auditory information is integrated with visual information to increase our ability to detect a visual stimulus. However, these results are limited because they incorporated simple visual sequences as a stimulus (graphical images or patterns). In real life, the stimulus may be more complex and information can be derived from the complicated context of the stimulus. Thus, our results are novel because we used a complex stimulus similar to stimuli experienced in real life. We found that the added auditory information enhances visual information about the rotational motion of the stimulus (only visual information is not sufficient), which is supported by previous studies (Wuerger et al. 2003; Beauchamp et al. 2004; Lewis and Noppeney 2010). For example, the study by Lewis and Noppeney (2010) indicated that synchronized visual and auditory information facilitated motion discrimination under insufficient visual conditions. Specifically, the synchronized visual and auditory information may amplify visual information processing by increasing the connectivity between early visual and auditory areas in the brain (Lewis and Noppeney 2010). It is also suggested that synchronized visual and auditory information are processed independently in the visual and auditory pathways and then combined at a 
decision level (Wuerger et al. 2003). In summary, these findings demonstrate that added auditory information to visual information can enhance the ability of an individual to accurately identify the rotational motion of a complex visual stimulus.

\section{Considerations}

Our results are limited to the stimuli presented using the videos. We measured the prediction accuracy responding to the videos projected on a screen, so it is not clear whether such prediction accuracy is also shown from the participants witnessing live situations. In addition, our findings are obtained from table-tennis service, therefore, it is not clear whether these findings will generalize to other situations. Future studies, therefore, are needed to examine the prediction accuracy for direction and rotational motion based on visual and auditory information in other situations such as walking or driving. Furthermore, not measuring reaction time in our study is a limitation of the study because the duration for prediction in sports is critical. Thus, future studies is needed to identify the effect of visual and auditory information on reaction time and the relation between reaction time and prediction accuracy. Moreover, healthy young expert players participated in this study. Therefore, it is not clear whether our findings will apply to other populations such as children, older adults, or adults with neurological disorders. Future studies, therefore, are needed to examine the prediction accuracy for direction and rotational motion in other populations.

In summary, we provide evidence that additional visual information can enhance the ability to identify the direction of a stimulus, whereas added auditory information to visual information can enhance the ability to identify the rotational motion of a stimulus. 
Our findings are the first in the motor control literature to show that additional auditory information to visual information can enhance the ability to identify the rotational motion of a complex visual stimulus. Identifying the rotational motion of a visual stimulus can be critical in real life, such as estimating the motion of a falling rock while hiking. Therefore, training protocols that attempt to optimize motion estimation, such as in sports training, will benefit from adding sound to the visual stimulus.

\section{Conflict of interest statement}

The authors declare that there are no conflicts of interest.

\section{Acknowledgement}

The authors would like to acknowledge Dr. Neha Lodha for her comments on the manuscript. This CRI work was supported by the National Research Foundation of Korea (NRF) grant funded by the Korea government (MEST) (NRF-2010-0021103). 


\section{REFERENCE}

Abernethy, B. and Russell, D.G. 1987. The Relationship between Expertise and Visual-Search Strategy in a Racquet Sport. Human Movement Science 6: 283-319. doi:Doi 10.1016/01679457(87)90001-7

Beauchamp, M.S., Lee, K.E., Argall, B.D., and Martin, A. 2004. Integration of Auditory and Visual Information about Objects in Superior Temporal Sulcus. Neuron 41: 809-823. doi:http://dx.doi.org/10.1016/S0896-6273(04)00070-4

Buchtel, H.A., Butter, C.M., and Ayvasik, B. 1996. Effects of stimulus source and intensity on covert orientation to auditory stimuli. Neuropsychologia 34: 979-985.

Deery, H.A. 2000. Hazard and risk perception among young novice drivers. Journal of safety research 30: 225-236.

Desantis, A., Mamassian, P., Lisi, M., and Waszak, F. 2014. The prediction of visual stimuli influences auditory loudness discrimination. Experimental Brain Research 232: 3317-3324.

Frassinetti, F., Bolognini, N., and Làdavas, E. 2002. Enhancement of visual perception by crossmodal visuo-auditory interaction. Experimental Brain Research 147: 332-343.

Georgopoulos, A.P., Kalaska, J.F., Caminiti, R., and Massey, J.T. 1982. On the relations between the direction of two-dimensional arm movements and cell discharge in primate motor cortex. The Journal of Neuroscience 2: 1527-1537.

Gibson, J.J. 1957. Optical motions and transformations as stimuli for visual perception. Psychological Review 64: 288.

Hol, K. and Treue, S. 2001. Different populations of neurons contribute to the detection and discrimination of visual motion. Vision research 41: 685-689.

Lewis, R. and Noppeney, U. 2010. Audiovisual synchrony improves motion discrimination via enhanced connectivity between early visual and auditory areas. The Journal of neuroscience 30: 12329-12339. 
Lippert, M., Logothetis, N.K., and Kayser, C. 2007. Improvement of visual contrast detection by a simultaneous sound. Brain research 1173: 102-109.

Meyer, G. and Wuerger, S. 2001. Cross-modal integration of auditory and visual motion signals. Neuroreport 12: 2557-2560.

Noesselt, T., Bergmann, D., Hake, M., Heinze, H.-J., and Fendrich, R. 2008. Sound increases the saliency of visual events. Brain Research 1220: 157-163.

doi:10.1016/j.brainres.2007.12.060

Olivers, C.N. and Van der Burg, E. 2008. Bleeping you out of the blink: Sound saves vision from oblivion. Brain research 1242: 191-199.

Saunders, J.A. and Knill, D.C. 2003. Humans use continuous visual feedback from the hand to control fast reaching movements. Experimental Brain Research 152: 341-352.

Soto-Faraco, S., Spence, C., and Kingstone, A. 2005. Assessing automaticity in the audiovisual integration of motion. Acta psychologica 118: 71-92.

Treue, S., Hol, K., and Rauber, H.-J. 2000. Seeing multiple directions of motion-physiology and psychophysics. Nature neuroscience 3: 270-276.

Underwood, G., Chapman, P., Bowden, K., and Crundall, D. 2002. Visual search while driving: skill and awareness during inspection of the scene. Transportation Research Part F: Traffic Psychology and Behaviour 5: 87-97.

Vroomen, J. and Gelder, B.d. 2000. Sound enhances visual perception: Cross-modal effects of auditory organization on vision. Journal of Experimental Psychology: Human Perception and Performance 26: 1583-1590. doi:10.1037/0096-1523.26.5.1583

Welch, R.B., DutionHurt, L.D., and Warren, D.H. 1986. Contributions of audition and vision to temporal rate perception. Perception \& Psychophysics 39: 294-300.

Wuerger, S., Hofbauer, M., and Meyer, G. 2003. The integration of auditory and visual motion signals at threshold. Perception \& Psychophysics 65: 1188-1196. 


\section{FIGURE LEGENDS}

Figure 1. Experimental setup. Lateral view of the experimental set up. The video of the service was projected on a screen $3 \mathrm{~m}$ away from the participants. In some condition, the sound of the racquet hitting the ball was provided via a speaker ( $2 \mathrm{~m}$ away from the participants; $50 \mathrm{~dB}$ measured from $30 \mathrm{~cm}$ away). Participants indicated the expected direction and rotational motion of service on the keyboard, which was located on a desk in front of them.

Figure 2. Experimental conditions. There were five types of videos that reflected the experimental conditions ( 5 experimental conditions $\times 12$ trials).

Figure 3. Prediction accuracy with visual information A) Prediction accuracy for direction tended to increase with more visual information. B) Prediction accuracy for rotational motion of service was not influenced by visual information.

Figure 4. Prediction accuracy with visual and auditory information. A) Prediction accuracy for direction was not improved with the addition of auditory information to visual information. B) Prediction accuracy for rotational motion of service increased with the addition of auditory information to visual information. 


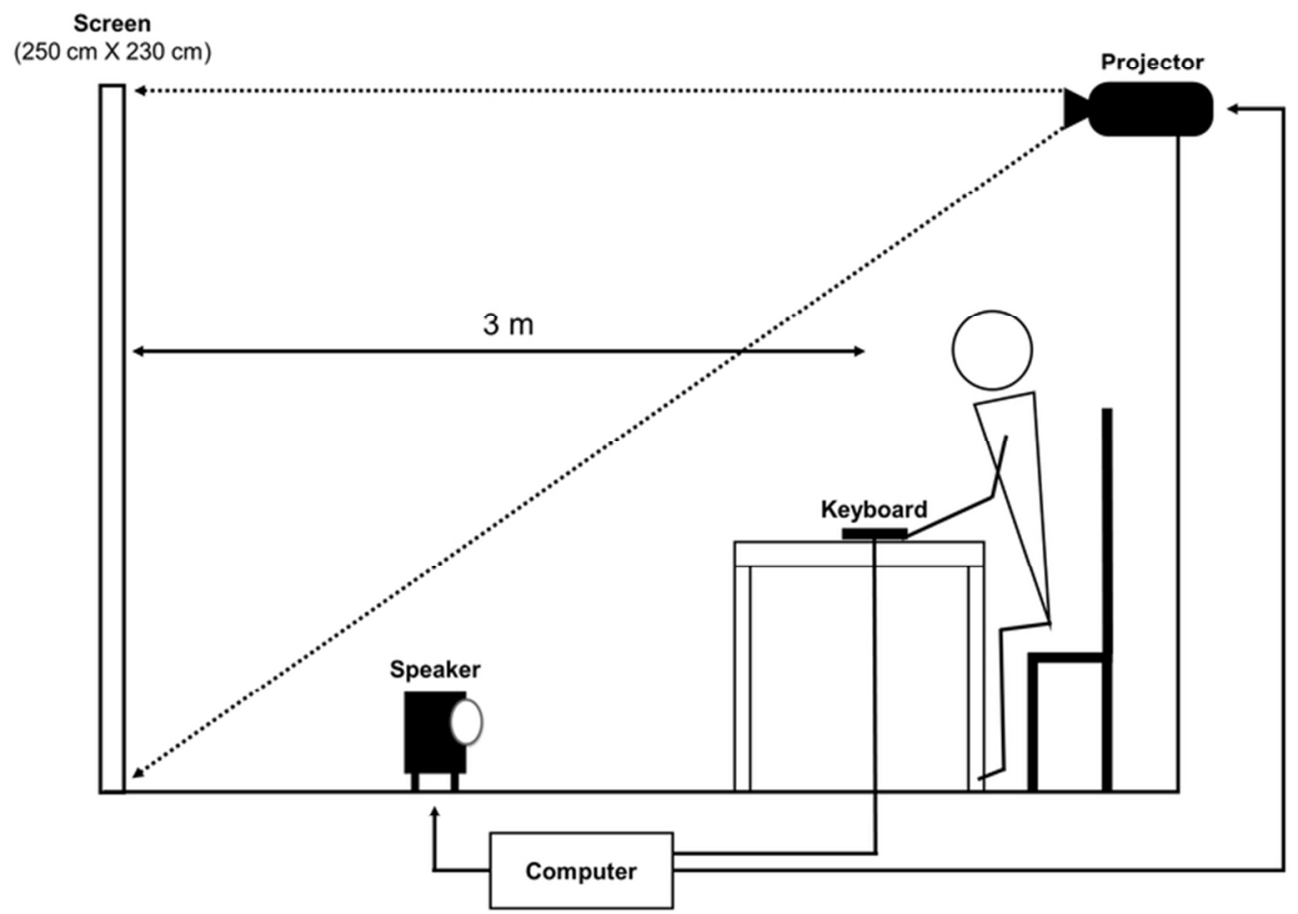

Figure 1. Experimental setup. Lateral view of the experimental set up. The video of the service was projected on a screen $3 \mathrm{~m}$ away from the participants. In some condition, the sound of the racquet hitting the ball was provided via a speaker ( $2 \mathrm{~m}$ away from the participants; $50 \mathrm{~dB}$ measured from $30 \mathrm{~cm}$ away). Participants indicated the expected direction and rotational motion of service on the keyboard, which was located on a desk in front of them. $66 \times 50 \mathrm{~mm}(300 \times 300 \mathrm{DPI})$ 


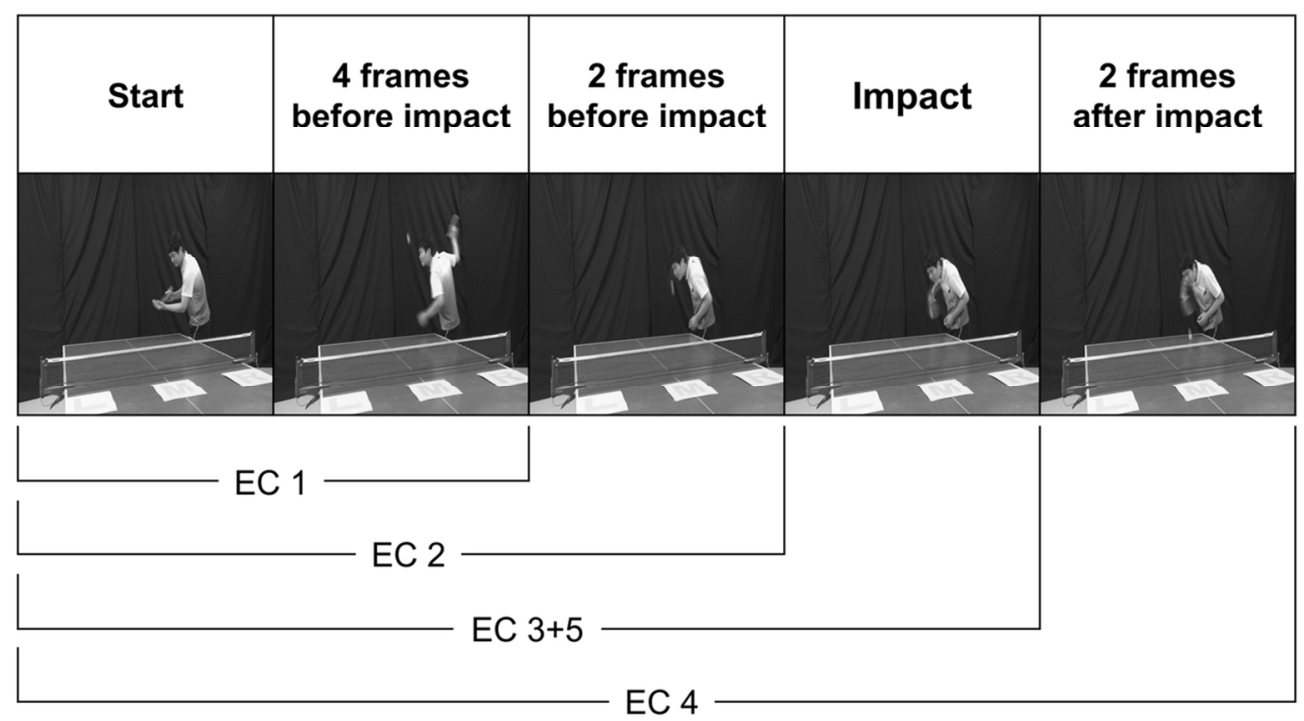

Figure 2. Experimental conditions. There were five types of videos that reflected the experimental conditions (5 experimental conditions $\times 12$ trials). $109 \times 65 \mathrm{~mm}(300 \times 300 \mathrm{DPI})$ 

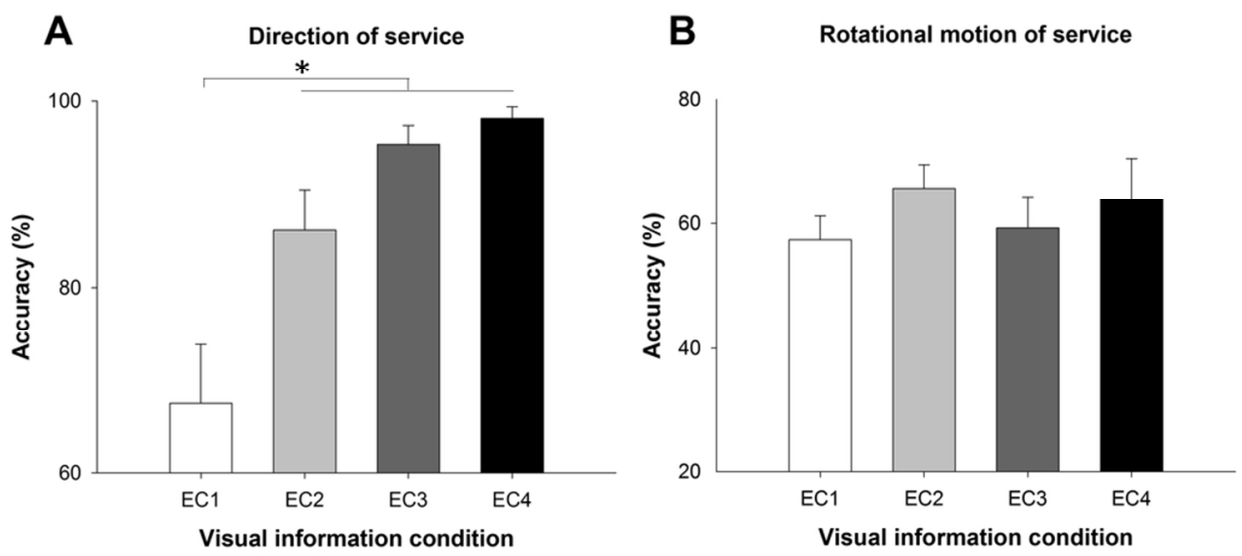

Figure 3. Prediction accuracy with visual information A) Prediction accuracy for direction tended to increase with more visual information. B) Prediction accuracy for rotational motion of service was not influenced by visual information.

$91 \times 45 \mathrm{~mm}(300 \times 300 \mathrm{DPI})$ 

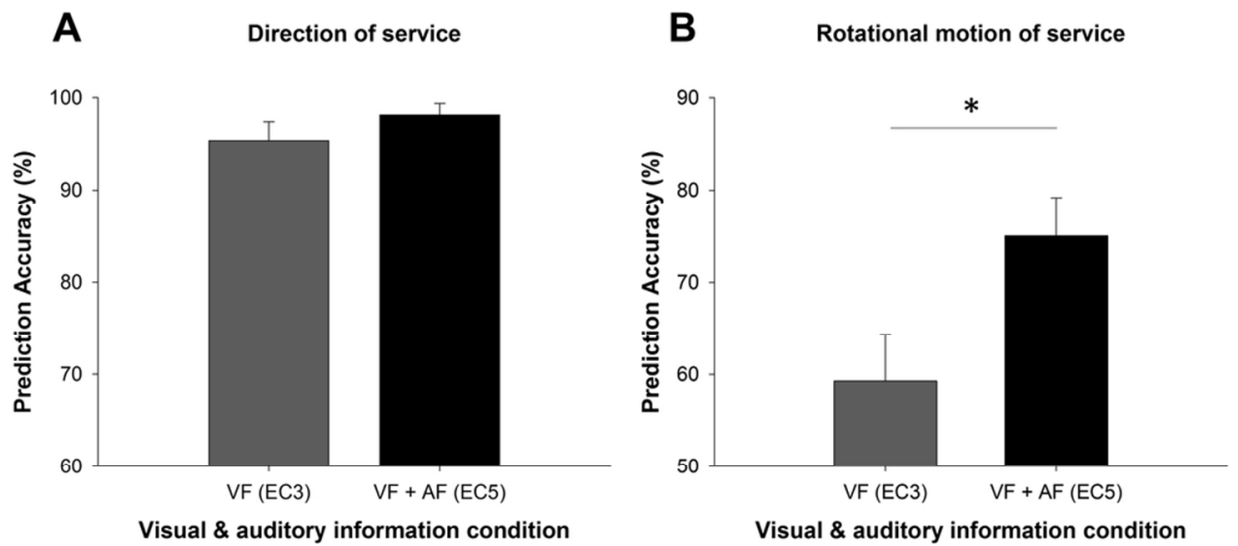

Figure 4. Prediction accuracy with visual and auditory information. A) Prediction accuracy for direction was not improved with the addition of auditory information to visual information. B) Prediction accuracy for rotational motion of service increased with the addition of auditory information to visual information. $91 \times 45 \mathrm{~mm}(300 \times 300 \mathrm{DPI})$ 